\title{
Phase Registration Improves Classification and Clustering of Cycles Based on Self-Organizing Maps
}

\author{
Juan-Carlos Quintana-Duque \\ University of Konstanz \\ Universitätsst. 10, 78464. Konstanz, Germany. \\ juan.quintana@uni-konstanz.de
}

\author{
Dietmar Saupe \\ University of Konstanz \\ Universitätsst 10, 78464. Konstanz, Germany. \\ dietmar.saupe@uni-konstanz.de
}

\begin{abstract}
Self-Organizing Maps (SOMs), also known as SelfOrganizing Feature Maps, have been used to reduce the complexity of joint kinematic and kinetic data in order to cluster, classify and visualize cyclic motion data. In this paper we describe the results after training SOMs with preprocessed data based on phase registration by dynamic time warping. For validation, we recorded acceleration data of human locomotion varying the treadmill slope, activity (i.e., walking, jogging, running), and whether or not $1.5 \mathrm{~kg}$ weights were attached to the ankles. The topological quality of the SOMs after training improved when the phase registration was applied. Furthermore, test (i.e., combination of treadmill slope and type of gait) and subject classification improved, in particular for walking data, when the phase registration was applied for each individual activity. Activity classification improved when the phase registration was calculated from all cycles of our experiments together.
\end{abstract}

\section{Author Keywords}

Acceleration; Classification; Clustering; Phase registration; Gait Analysis; SOM.

\section{ACM Classification Keywords}

H.3.3. Information Storage And Retrieval: Information Search and Retrieval

\section{INTRODUCTION}

Cyclic motion is at the core of many sports such as running, swimming, or cycling. The study of corresponding kinematic variables is fundamental for the evaluation of training routines and the assessment of performance. Frequently, the analysis of recorded cycles is performed manually by experts. However, their conclusions may not agree because of the difficulty in comprehending large amounts of information or because different levels of expertise might lead to different interpretations. There is therefore a clear need for a method which overcomes these two limitations and enables large quantities of gait data to be analysed and interpreted objectively [1].

Permission to make digital or hard copies of all or part of this work for personal or classroom use is granted without fee provided that copies are not made or distributed for profit or commercial advantage and that copies bear this notice and the full citation on the first page. Copyrights for components of this work owned by others than ACM must be honored. Abstracting with credit is permitted. To copy otherwise, or republish, to post on servers or to redistribute to lists, requires prior specific permission and/or a fee. Request permissions from permissions@ acm.org.

WOAR'15, June 25 - 26, 2015, Rostock, Germany.

Copyright (C) 2015 ACM ISBN 978-1-4503-3454-9/15/06..\$15.00.

DOI: http://dx.doi.org/10.1145/2790044.2790053
In order to provide a tool for cycle registration that facilitates the analysis of cycles in cyclical motion data, we proposed in [12] a method called eDBA, which calculates the average of a set of cycles based on dynamic time warping (DTW) and a modification of DTW barycentric averaging (DBA). The eDBA algorithm allows the study of features of kinematic variables in cyclic motion depending on the phase using the eDBA average cycle as reference for phase registration.

Clustering, classification and visualization of high dimensional data collected from motion (e.g., kinematic, kinetic and electromyographic data) can be based on Self-Organizing Maps (SOMs), described in [7]. SOMs reduce the complexity of high dimensional data to usually two-dimensional regular spacing grids. This representation can be easily interpreted by humans, e.g., visualization using SOMs was done for gait stem-patterns in [1] and for young-old gait patterns in [2]. Furthermore, one can build models on top of the SOMs, e.g., recognition of gait patterns [9] and of emotions in gait [5]. Typically, these models are simple local or nearest-neighbor models.

Many publications are devoted to classification of cyclic motion with SOMs but there are not yet any studies on the effects of registration of cycles on the quality of the SOMs, and whether there is any benefit in doing this. In this contribution, we compared the quality and the classification performance of the trained SOMs with and without data preprocessing using the phase registration of cycles.

\section{METHODS}

\section{Self-Organizing Maps (SOMs)}

The Self-Organizing Map (SOM) is an unsupervised neural network algorithm which implements nonlinear projection of multi-dimensional data onto a low dimensional array of vectors, i.e., a finite set of model vectors. The low-dimensional discretized representation of the input space of the training samples is called a map that should represent meaningful entities in the data.

Each model vector of the SOM is determined as a weighted average of all of those training vectors that are mapped into the topological neighborhood around the corresponding model [8]. Thus, self-organizing maps learn both the distribution and topology of the input vectors they are trained on.

For SOMs it is assumed that a map with a sufficient number of neurons conveys information about input data sets in a clear manner. The right selection of the parameters of the 

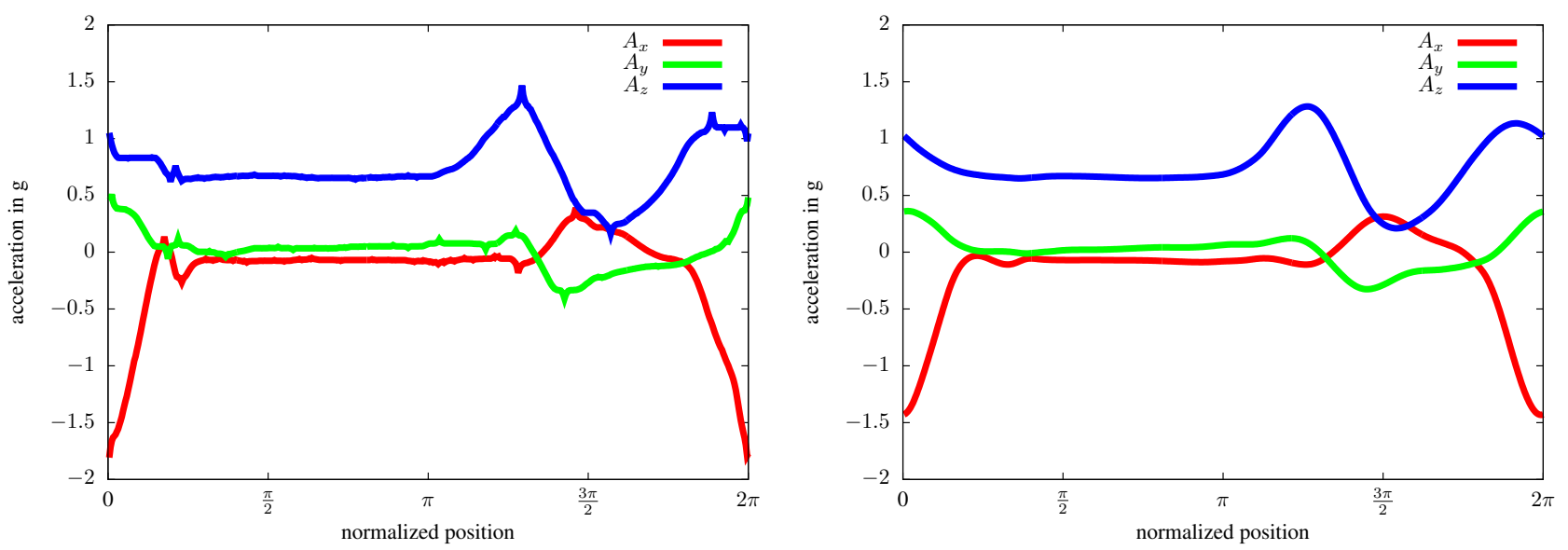

Figure 1. Average results of 3D walking acceleration data, i.e., $\left(A_{x}, A_{y}, A_{z}\right)$ of a sensor placed on the foot, using DBA (left) and eDBA (right).

map (e.g., the number of neurons, dimensions of the map grid, map lattice and shape) is a key issue due to the trade off between the representation accuracy and the avoidance of over-fitting. Normally, different sets of parameters are tested and the quality of the resulting maps after training is evaluated to select the best one. Some of the quality measures are explained in the next section.

\section{SOM Quality}

After the SOM has been trained, it is important to know whether it has properly adapted itself to the training data. Map quality measures have been proposed for evaluating the mapping precision, the topology preservation or a combination of both. Some of them are described in [10] based on the closest model vector in the map to an input vector, i.e., the Best Matching Unit (BMU).

In this paper, the maps and three quality measures were calculated using the Matlab toolbox provided in [14]. The quality measures were the following: 1) The mapping precision was measured using average quantization error between data vectors and their BMUs on the map. 2) The topological representation accuracy was measured as the percentage of data vectors for which the first- and second-BMUs are not adjacent units. 3) The average 'combined' error [6] over all input vectors was calculated from the sum of quantization error (i.e., accuracy of the map in representing the input data set) and topographic error (i.e., if the map is defined by means of a graph, where the edge lengths are the distances between nodes and the graph nodes are the model and input vectors, then the topographic error is computed using Dijkstra's algorithm [4] from the input vector $x$ to the second-BMU passing by the first-BMU).

\section{DBA and modifications of DBA}

For a set of time series a characteristic 'average' time series may be desired. DTW barycentric averaging (DBA) [11] is a local optimization algorithm, similar to clustering methods like k-means, which minimizes the total cost given by the sum of all costs for the DTW alignments with the average series. In each iteration of the algorithm all sample points of the average series are updated by the arithmetic mean of all corresponding samples of the given series.

Applying the standard cost function in the DTW algorithm in the context of the DBA method yields sharp peaks of the average cycle that are not characteristic of the underlying motion but can be regarded as DBA artifacts. See the left plot in Fig. 1 for an example of the results applying DBA. The cause for this problem is that in the averaging step of DBA, many samples of a given cycle may be aligned with the same sample of the average cycle. This strongly pulls the sample of the average cycle towards the given cycle, yielding the spurious peaks.

Our solution eDBA described in [12], introduces weights for the averaging such that each cycle is accounted for by the same weight. See the right plot in Fig. 1 for an example of results applying eDBA. Moreover, consecutive samples aligned with the same sample of the average cycle lead to parts of piecewise constant phase, not appropriate for physical motion. Thus, we include a regularization term in the cost function to penalize constant phase,

$$
c(i, j)=(1-\lambda) \frac{\left\|x_{a}[i]-x_{k}[j]\right\|^{2}}{E}+\lambda \frac{(i-j)^{2}}{T}
$$

where $x_{a}[i]$ is a point in the average, $x_{k}[j]$ is a point in the cycle $k, E$ and $T$ are normalization constants, such that for $\lambda=0.5$ on average the two terms contribute roughly the same costs in the DTW algorithm. We used $E=$ $\frac{1}{N} \sum_{n=0}^{N-1}\left\|x_{a}[n]\right\|^{2}$ and $T=\frac{N}{4}$. The parameter $\lambda \in[0,1]$ determines the trade off between the classical DBA $(\lambda=0)$ and plain arithmetic averaging $(\lambda=1)$.

\section{Phase registration using average eDBA}

After the computation of the average cycle, all cycles, given by time series $x_{k}[j]$, can be registered by DTW with the average cycle so that each sample $x_{k}[j]$ is attributed by the phase of the corresponding point $x_{a}[j]$ on the average cycle. 

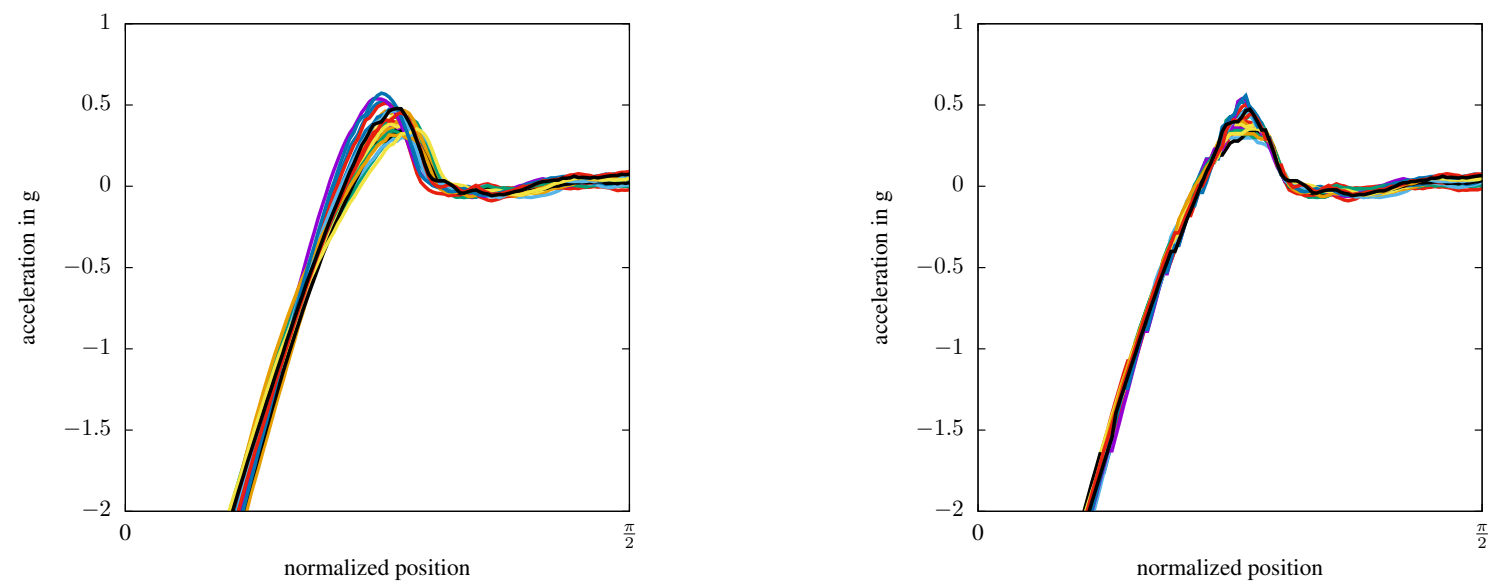

Figure 2. Small set of $A_{x}$ cycles without aligned (left plot) and the aligned, time warped cycles (right plot).

For each phase value $\phi=2 \pi i / N, i=0,1, \ldots, N-1$ let the set $A_{\phi}$ denote the set of all points from the entire motion trajectory assigned to the phase $\phi$. By design of the DTW algorithm the sets $A_{\phi}$ need not be disjoint and may contain several points of a single cycle $x_{k}[n]$. Such cases are artifacts resulting in physically unrealistic phase discontinuities and time intervals of constant phase, respectively. Fig. 2 shows the effectiveness of the time warping of a small set of cycles for the first quarter of the normalized phase. The warped series are much closer together.

\section{EXPERIMENTAL SETUP}

Eight healthy adults ( 7 male, 1 female, age $26 \pm 2.6$ years old, weight $62 \pm 7.2 \mathrm{~kg}$ ) did 12 tests of 3 minutes each on a treadmill (walking, jogging, running). The protocol of the tests is given in Table 1. We varied the treadmill slope, speed, and whether or not $1.5 \mathrm{~kg}$ weights were attached to the ankles. Before the recording session, subjects practiced for a few minutes on the treadmill at different speeds in order to get familiar with the device. The test sequence was identical for all subjects. One minute of rest was given after 9 minutes of tests.

\begin{tabular}{|c|c|c|c|c|c|}
\hline no. & activity & time & speed & slope & weights \\
\hline 1 & walking & $3 \mathrm{~min}$ & $2.5 \mathrm{~km} / \mathrm{h}$ & $0 \%$ & - \\
2 & walking & $3 \mathrm{~min}$ & $2.5 \mathrm{~km} / \mathrm{h}$ & $5 \%$ & - \\
3 & walking & $3 \mathrm{~min}$ & $2.5 \mathrm{~km} / \mathrm{h}$ & $10 \%$ & - \\
& rest & $1 \mathrm{~min}$ & & & \\
4 & jogging & $3 \mathrm{~min}$ & $5.0 \mathrm{~km} / \mathrm{h}$ & $0 \%$ & - \\
5 & jogging & $3 \mathrm{~min}$ & $5.0 \mathrm{~km} / \mathrm{h}$ & $5 \%$ & - \\
6 & jogging & $3 \mathrm{~min}$ & $5.0 \mathrm{~km} / \mathrm{h}$ & $10 \%$ & - \\
& rest & $1 \mathrm{~min}$ & & & \\
7 & running & $3 \mathrm{~min}$ & $7.5 \mathrm{~km} / \mathrm{h}$ & $0 \%$ & - \\
8 & running & $3 \mathrm{~min}$ & $7.5 \mathrm{~km} / \mathrm{h}$ & $5 \%$ & - \\
9 & running & $3 \mathrm{~min}$ & $7.5 \mathrm{~km} / \mathrm{h}$ & $10 \%$ & - \\
& rest & $1 \mathrm{~min}$ & & & \\
10 & walking & $3 \mathrm{~min}$ & $2.5 \mathrm{~km} / \mathrm{h}$ & $0 \%$ & $3 \mathrm{~kg}$ \\
11 & walking & $3 \mathrm{~min}$ & $2.5 \mathrm{~km} / \mathrm{h}$ & $5 \%$ & $3 \mathrm{~kg}$ \\
12 & walking & $3 \mathrm{~min}$ & $2.5 \mathrm{~km} / \mathrm{h}$ & $10 \%$ & $3 \mathrm{~kg}$ \\
\hline
\end{tabular}

Table 1. Test protocol for each subject
The motion data was acquired by a 3D accelerometer (RehaWatch, Hasomed), attached to the right shoe. The fastening of the sensor to the shoe was done by a special foot mounting with velcro. The sensor yielded time series $A_{x}, A_{y}, A_{z}$ of acceleration (in units of $\mathrm{g}$, acceleration by gravity) at a sampling rate of $600 \mathrm{~Hz}$.

\section{DATA PROCESSING}

The noise in the 3D acceleration data was reduced by a Savitzky-Golay filter [13], i.e., by least squares regression on a sliding window with quadratic polynomials and a window of 91 samples, yielding a filter with a cutoff frequency of 12 $\mathrm{Hz}$.

The gait cycles could clearly be segmented by extremal values in $A_{x}$, designating the instant of heel strike in each cycle. Fig. 3 shows an example of the segmentation points for each cycle. In this figure and in all collected data, the cycles have different amplitude and duration. Then, each cycle was Fourier transformed and upsampled by trigonometric interpolation to 512 samples per cycle. The first 20 cycles of each test were discarded to remove the transition from stance to swing motion.

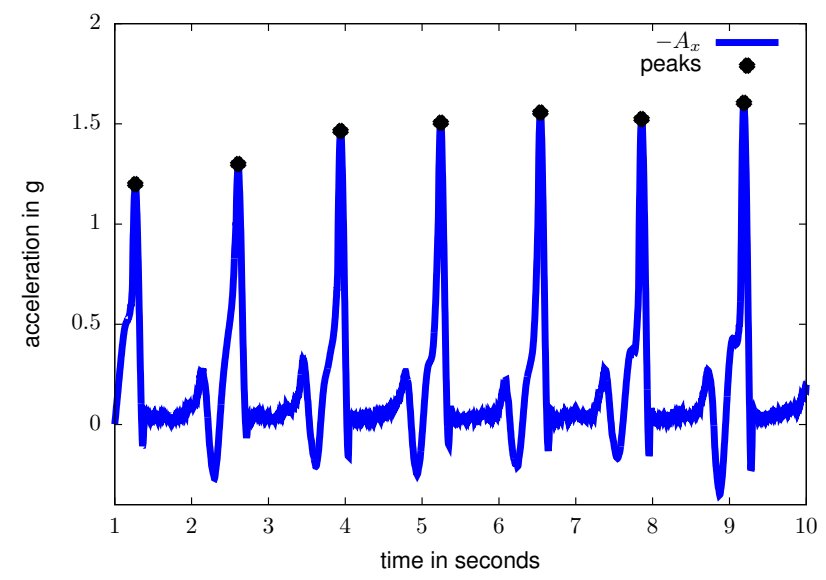

Figure 3. Segmentation of cycles 

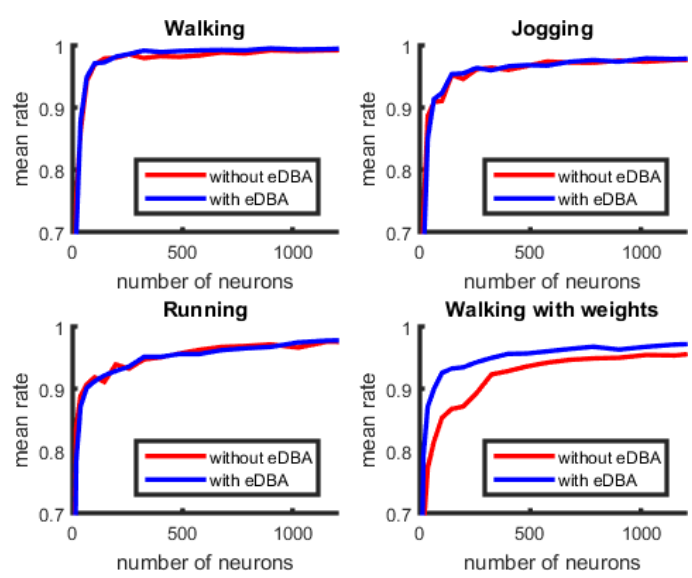

Figure 4. Mean rate of subject classification success for each activity after training with and without phase registration in preprocessing step.
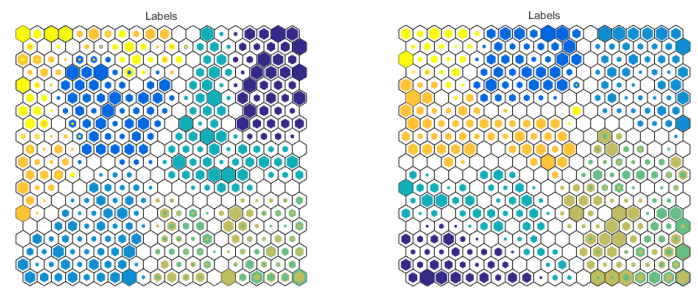

Figure 5. Example of subject classification in a $20 \times 20$ map of cycles obtained from the activity walking with weights after training with (right side) and without (left side) phase registration in preprocessing step. Each of the eight colors corresponds to one subject.

For each single gait activity (i.e., walking without weights, jogging, running, walking with weights) the average eDBA was calculated with $\lambda=0.5$. The cycles of each activity were registered using their corresponding average eDBA. Furthermore, in order to test the effects of a unique average for all activities we calculated the average eDBA of all activities together and the cycles were registered with this average.

Cycle classification was performed using SOM with sizes $n \times n$ and $n=2,4, \ldots, 40$. The number of neurons and their topological relations were fixed from the beginning of each training: 2-dimensional map with hexagonal lattice and a sheet form was used for all tests (i.e., the map sides were not connected to each other). Before the training, initial values were given to the model vectors by a linear initialization. The training was done with a batch algorithm. The input data was composed by the concatenated acceleration vectors $\left(A_{x}, A_{y}, A_{z}\right)$ of each cycle.

\section{RESULTS AND DISCUSSION}

In the following, we compared the classification performance, the representation accuracy and the topological quality of each SOM as a function of the number of neurons in the SOMs. We trained the SOMs using the acceleration data from each single activity separately (i.e., walking without weights, jogging, running, walking with weights) and together.

\section{Classification performance}

The mean success rate of subject classification and test classification are shown in Fig. 4 and Fig. 6, respectively. On the
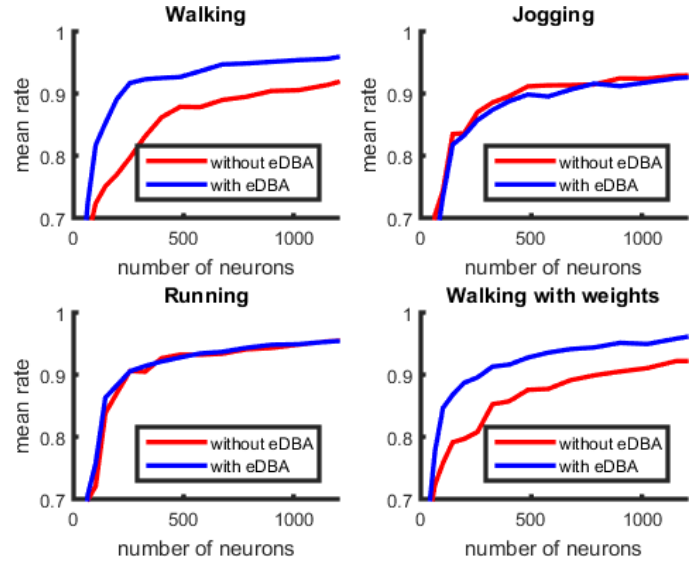

Figure 6. Mean rate of test classification success for each activity after training with and without phase registration in preprocessing step.
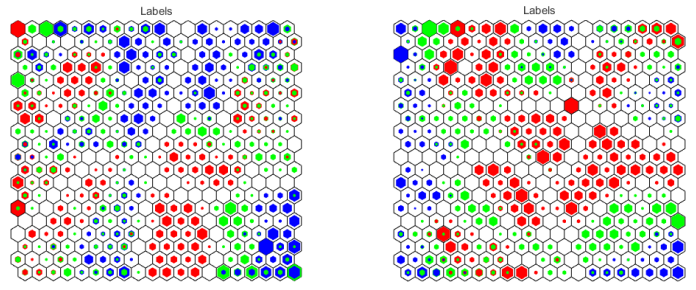

Figure 7. Example of test classification in a $20 \times 20$ map of cycles obtained from the activity walking with weights after training with (right side) and without (left side) phase registration in preprocessing step. Each of the three colors corresponds to one slope.

one hand, the results of subject classification ranged between 0.9 and 1 when the number of neurons in the map exceeded 400. The results did not improve when the phase registration was used. On the other hand, the results of test classification ranged between 0.8 and 1 when the number of neurons in the map exceeded 400. The results improved in particular for walking data (i.e., with and without weights).

Walking, jogging and running are generally considered as distinct gait modes with different mechanics and energetics. Walking gait may be defined by the existence of a double support phase during stance, whereas running has a 'flight' phase during which neither limb is in ground contact [3]. The larger the contact between the foot and the treadmill, the more irregular the motion pattern is.

Walking patterns were more irregular than jogging and running patterns, i.e., in our experiments the coefficient of variation of step duration of walking was larger than running and jogging for all subjects. Thus, our improvement of classification performance by cycle alignment was better for activities containing a larger degree of variability, which was to be expected. The test protocol described in Table 1 was not randomized for each user. However, with test randomization we would expect better results because the motion pattern recorded in each test would appear more irregular due to changes of gait speed between consecutive tests.

Fig. 5 and Fig. 7 show the hit histogram for each neuron in a $20 \times 20$ SOM trained with acceleration data obtained from walking data with weights. Hit histograms indicate how the 
best matching units of some data sets are distributed on a map. In each figure, regions with the same labels are distinguishable. In Fig. 5 regions corresponding to subjects and in Fig. 7 small regions corresponding to the tests performed by subjects (i.e., for this example the three slopes) are discernible in the map. The map clustered the tests together for each subject. This indicates that the variability between subjects was larger than the variability of the tests of each subject.

Finally, we observed the effects of phase registration calculating the eDBA average from the cycles of all tests together (i.e., 12 tests given in Table 1). The SOMs were trained for the same size as before with and without phase registration in the preprocessing step. Due to limited amount of space, we cannot show the results here. The subject classification ranged from 0.9 to 0.95 did not improve, but the test classification and activity classification (i.e., walking, jogging, running, walking with weights) improved slightly. The rate of test classification ranged from 0.7 to 0.86 improved $0.005-$ 0.01 , and the rate of activity classification ranged from 0.9 to 0.95 improved $0.005-0.02$.

\section{SOM quality}

Fig. 8 shows the mean quantization error with and without registration based on eDBA averaging in the preprocessing step against the number of neurons in the map. Fig. 9 shows the combined error explained previously against the number of neurons in the map. The quantization and the combined errors were normalized by the norm of each input vector.

The mean quantization and the combined error curves are decreasing with the number of neurons in the map. Furthermore, the normalized error values with eDBA in the preprocessing step were smaller than without registration for walking with and without weights. Starting from the lowest number of neurons, when the number of neurons was incremented the error curves fell sharply until a bend is achieved. Then, the error curves decreased gently. The error values and the position of the bend at the error curves are relevant to select the appropriated number of neurons of the map, i.e., avoiding overfitting while representing the input data distribution.

For example, if the bend in the curve is defined as the bisection point for which the sum of the least square errors of the fitting of two lines to all the points on the left and right sides is minimal, then the bend points in Fig. 8 and Fig. 9 are located at 36-196 neurons.

For data mining and visualization applications it is important that two neighboring neurons represent the same type of data and two distant neurons (according to the grid) represent different data. In Fig. 10 we plotted the percentage of data vectors for which the first and second BMUs are non-adjacent units against the number of neurons in the map. This percentage was smaller for maps trained with phase registration based on average eDBA in the preprocessing step except for walking. For walking and maps with neurons less than 256, the percentage was higher than without phase registration, although the quantization error and the combined error were the lowest among the activities.
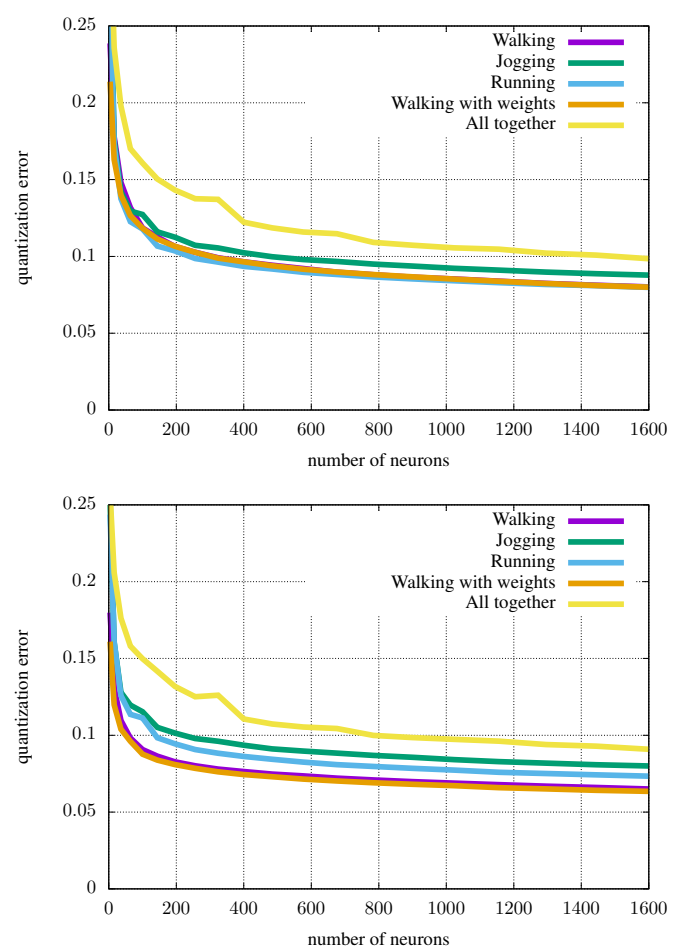

Figure 8. Mean quantization error without (top) and with (bottom) eDBA in preprocessing step
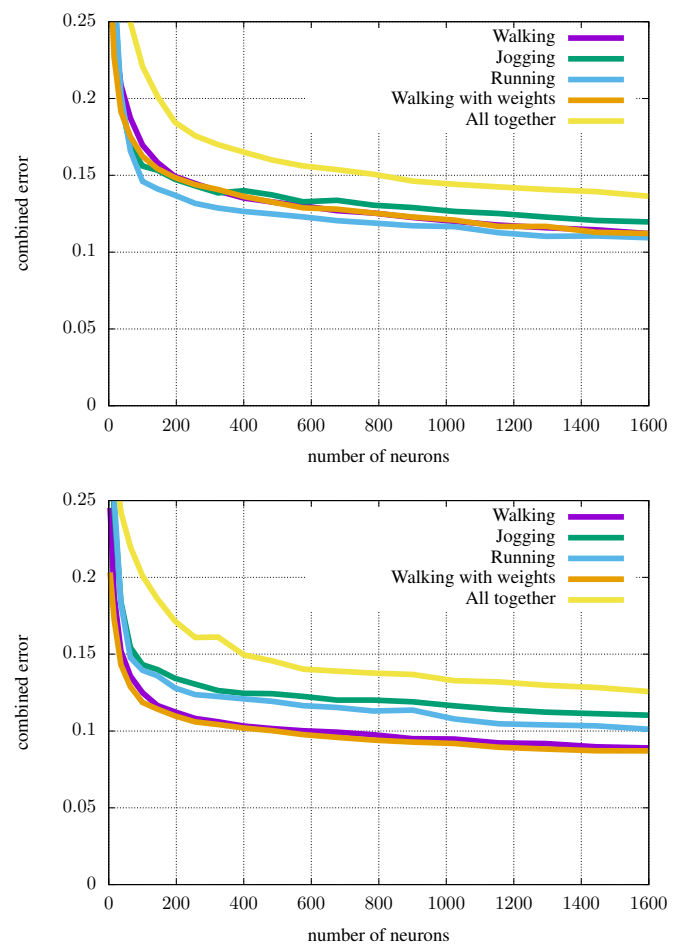

Figure 9. Mean combined error without (top) and with (bottom) eDBA in preprocessing step 

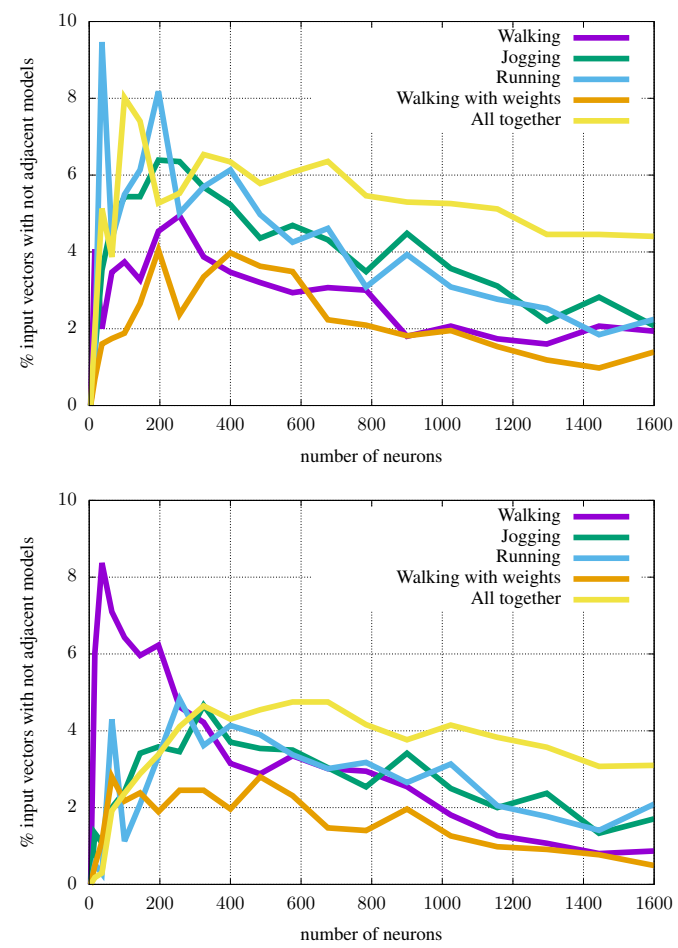

Figure 10. Percentage of input vectors for which first-and second-BMUs are not adjacent units in the resulting map without (top) and with (bottom) eDBA in preprocessing step

For all curves in Fig. 10, when the number of neurons increased the percentage grew until a peak was reached. Then, the percentage fell smoothly. The shape of the curves shows the trade-off between the size of a map and its fit to the given data. When the number of neurons was too small, topological errors were low but the quantization error was large as shown in Fig. 8. When the number of neurons increased, the topological errors decreased. The curves do not show overfitting of the SOMs on the data because the maximal number of neurons (i.e., 1600 neurons) was much smaller than the number of training samples.

\section{CONCLUSION}

We tested SOMs of different size and compared the resulting maps using three quality measures based on mapping precision, topology preservation and a combination of both. The phase registration as a part of the preprocessing steps increased the mapping precision and the topology preservation quality. Furthermore, the phase registration improved the success rate of test classification especially for walking gait (e.g., with 100 neurons or more the improvement was 5\%-10\%).

From our results, we suggest to apply phase registration of the cycles with eDBA for each activity, in particular when cycle classification is required (e.g., with different slopes). For activity classification (i.e., walking, jogging, running) the registration using eDBA of all activities is recommended.

For the future work, we will develop a framework to validate the clustering results with experts and extend our approach of curve registration to continuous time. In addition, we will test the effects on the SOMs with different parameters for calculating the eDBA average and the phase registration. Furthermore, we will record more subjects in order to perform significance tests on our results.

\section{REFERENCES}

1. Barton, G., Lees, A., Lisboa, P., and Attfield, S. Visualisation of gait data with Kohonen self-organising neural maps. Gait \& Posture 24, 1 (2006), 46-53.

2. Begg, R., and Kamruzzaman, J. A comparison of neural networks and support vector machines for recognizing young-old gait patterns. In TENCON, vol. 1 (2003), 354-358.

3. Cappellini, G., Ivanenko, Y. P., Poppele, R. E., and Lacquaniti, F. Motor patterns in human walking and running. Journal of Neurophysiology 95, 6 (2006), 3426-3437.

4. Dijkstra, E. W. A note on two problems in connexion with graphs. Numerische Mathematik 1 (1959), 269-271.

5. Janssen, D., Schöllhorn, W. I., Newell, K. M., Jäger, J. M., Rost, F., and Vehof, K. Diagnosing fatigue in gait patterns by support vector machines and self-organizing maps. Human movement science 30, 5 (2011), 966-975.

6. Kaski, S., and Lagus, K. Comparing self-organizing maps. In ICANN. Springer, 1996, 809-814.

7. Kohonen, T. The self-organizing map. Proceedings of the IEEE 78, 9 (1990), 1464-1480.

8. Kohonen, T., Nieminen, I., and Honkela, T. On the quantization error in SOM vs. VQ: A critical and systematic study. In Advances in Self-Organizing Maps, vol. 5629. Springer Berlin Heidelberg, 2009, 133-144.

9. Koktas, N., Yalabik, N., and Yavuzer, G. Combining neural networks for gait classification. In Progress in Pattern Recognition, Image Analysis and Applications, vol. 4225. Springer Berlin Heidelberg, 2006, 381-388.

10. Mayer, R., Neumayer, R., Baum, D., and Rauber, A. Analytic comparison of self-organising maps. In Advances in Self-Organizing Maps, vol. 5629. Springer Berlin Heidelberg, 2009, 182-190.

11. Petitjean, F., Ketterlin, A., and Gançarski, P. A global averaging method for dynamic time warping, with applications to clustering. Pattern Recognition 44, 3 (2011), 678-693.

12. Quintana-Duque, J.-C., Saupe, D., and Vieten, M. Parametrization of cyclic motion and transversal sections. In Sportinformatik X (2014), 111-117.

13. Savitzky, A., and Golay, M. J. E. Smoothing and differentiation of data by simplified least squares procedures. Analytical Chemistry 36, 8 (1964), 1627-1639.

14. Vatanen, T., Osmala, M., Raiko, T., Lagus, K., Sysi-Aho, M., Oresic, M., Honkela, T., and Lähdesmäki, $\mathrm{H}$. Self-organization and missing values in SOM and GTM. Neurocomputing 147 (2015), 60 - 70. 